\title{
Effect of Huaier Granule and Transcatheter Arterial Chemoembolization on the Treatment of Advanced Liver Cancer
}

\author{
Zheng Yao \\ Zhejiang Cancer Hospital, Hangzhou 310000, Zhejiang, China \\ Email: 178461874@qq.com
}

\begin{abstract}
Objective - To explore the effect of Huaier granule combined with transcatheter arterial chemoembolization (TACE) in the treatment of advanced liver cancer. Methods — From January 2017 to January 2020, 150 patients with advanced liver cancer admitted to our hospital were selected as clinical subjects for a prospective non-randomized controlled study based on different treatment schedules. Among them, 50 patients were distributed to TACE group with TACE treatment alone. Besides, 50 patients were assigned to chemotherapy group and received systemic chemotherapy with Folfox 4 regimen alone. Among them, 50 patients were distributed as the observation group. The therapeutic effects of the three groups were compared. Results: The effective rate and disease control rate in observation group were significantly higher than those in TACE group and chemotherapy group $(\mathrm{P}<0.05)$. The rate of adr in observation group and TACE group was significantly lower than that in chemotherapy group $(\mathrm{P}<0.05)$. The survival rate of observation group was significantly higher than that of TACE group and chemotherapy group after 12 months follow-up $(\mathrm{P}<0.05)$. Conclusion — The combined treatment of huaier granule and TACE can improve the therapeutic effect and survival rate of patients with advanced liver cancer.

Keywords: advanced liver cancer, Huaier granule, transcatheter arterial chemoembolization (TACE)
\end{abstract}

Primary liver cancer (liver cancer) is a kind of cancer originating in human liver cells or intrahepatic bile duct epithelial cells. Among them, the group over 40 years old has a high incidence of liver cancer ${ }^{[1]}$. However, there are no obvious symptoms in the early stage of liver cancer, and most patients have developed to the advanced stage as diagnosed with adverse phenomena such as lung metastasis ${ }^{[2]}$. At this point, the patient has lost the opportunity of radical surgery, and the disease can be effectively controlled through other programs to inhibit the continuous growth of tumor lesions so as to alleviate the symptoms of the patient, and prolong the survival time of the patient. Transcatheter arterial chemoembolization (TACE) is an effective means for the clinical treatment of advanced liver cancer, which can promote the coagulation and necrosis of most tumor lesions ${ }^{[3]}$. However, most patients have residual tumor cells in their tumor lesions, so it is easy to relapse and metastasis, affecting the overall effect. Based on this, this paper mainly analyzed the effect of TACE combined with Huaier granule in the treatment of advanced liver cancer, as shown below.

\section{Data and methods}

\subsection{Clinical data}

From January 2017 to January 2020, 150 patients with advanced liver cancer admitted to our hospital were selected as clinical subjects for a prospective non-randomized controlled study based on different treatment regimenings. There were 33 males and 17 females in the TACE group, aged 30-69 years (49.72 \pm 14.36 ). There were 37 and 13 patients in TNM stage II and III, and 45 and 5 patients in child-pugh grade A and B. In the chemotherapy group, there were 32 males and 18 females, aged 31-69 years (50.02 14.43$), 38$ and 12 patients with TNM stage II and III, 43 and 7 patients with Child-Pugh grade A and B liver function. In the observation group, there were 35 males and 15 females, aged 31-68 years (49.86 \pm 14.40$), 37$ and 13 patients with TNM stage II and III, 42 and 8 patients with Child-pugh grade A and B liver function. Comparison of clinical data among the three groups showed no difference $(\mathrm{P}>0.05)$.

Inclusion criteria: (1) All patients demonstrated their willingness to cooperate the doctors to complete relevant examinations after admission, and the examination results showed that they met the liver cancer diagnostic criteria in the guidelines for the diagnosis and treatment of primary liver cancer of the ministry of health, PRC (2011 edition) ${ }^{[4]}$; (2) Measurable and definite lung metastatic lesions were present in all patients; (3) Liver function child-PughA grade or B grade, and no other anti-liver cancer treatment was performed before participating in the study; (4) Patients who voluntarily participate in the study, give informed consent to the study and sign relevant documents.

Exclusion criteria: (1) Patients with an expected survival time of about 3 months; (2) Patients aged 80 or under 18 years 
old; (3) Patients with early or intermediate liver cancer can be treated with surgery; (4) Patients receiving anti-liver cancer treatment such as immunotherapy and surgical treatment before participating in this study; (5) If the condition deteriorates, the treatment plan should be changed immediately or combined with other plans to treat the patient.

\subsection{Methods}

Patients in the TACE group received TACE treatment alone. Patients in chemotherapy group received systemic chemotherapy with Folfox4 regimen. The patients in observation group were treated with TACE and treated with huaier granule granule.

TACE procedures: Puncture the femoral artery of the patient with Selidinger technology, intubation was applied in the patient's liver proper artery, and the chemotherapy drugs (Oxaliplatin, Raltetrexed, etc.) should be infused. Then, the catheter was inserted into the patient's tumor supplying artery, and $50 \mathrm{mg}$ Oxaliplatin $+5-20 \mathrm{ml}$ ultra-liquefied lipiodol emulsion was injected to perform tumor vascular embolization. To ensure the embolization effect, gelatin sponge and Embosphere particle embolization agent can also be added to the emulsion to strengthen the embolization treatment. The duration of one interventional treatment lasted 4 weeks, and the patient underwent at least 2 cycles of treatment. After that, the frequency of subsequent TACE interventional therapy was determined in combination with the actual control of the patient's tumor lesions.

Chemotherapy scheme with Folfox4: On the first day of chemotherapy, Oxaliplatin (SFDA Approval Number H20031048, Shenzhen Neptunus Pharmaceutical Co., LTD.) was given for intravenous infusion of $85 \mathrm{mg} / \mathrm{m}^{2}$. On the first and second days of treatment, calcium folate (SFDA Approval Number H20010615, Chongqing YaoPharm., LTD.) was applied for $200 \mathrm{mg} / \mathrm{m}^{2}$ intravenous infusion, and the infusion time was controlled at $2 \mathrm{~h}$. Fluorouracil (SFDA Approval Number H31020593, Shanghai Sunrise Haipu Pharmaceutical Co., LTD.) was given with $400 \mathrm{mg} / \mathrm{m}^{2}$ intravenous injection and the dose of $600 \mathrm{mg} / \mathrm{m}^{2}$ of fluorouracil was maintained intravenously (the duration of infusion was $22 \mathrm{~h}$ ). The treatment was repeated every 2 weeks, with a cycle of 2 weeks and at least 4 cycles of treatment. After that, whether to continue chemotherapy or maintain dose chemotherapy or switch to other treatment regiments was considered in combination with the changes of patients' conditions.

Huaier granule (SFDA Approval Number Z20000109, Qidong Gaitianli Pharmaceutical Co., LTD.): administered after dissolved; after TACE intervention or systemic chemotherapy with Folfox 4 regimen for 3 days, the dose was controlled at $20 \mathrm{~g} / \mathrm{time}$ and the frequency was controlled at 3 times/day, and the medication was continued for 6 months.

\subsection{Observation indicators}

(1) The treatment effect of patients in the two groups in recent period: The changes of liver cancer tumor lesions after 2 months of treatment were determined through imaging examination, and the corresponding evaluation criteria were established ${ }^{[5]}$. Complete remission: If imaging shows no tumor lesion, there will be only cord-shaped image, which means that the tumor is completely gone and necrotic. Partial remission: If imaging shows that the tumor focus of the patient is reduced by more than $50 \%$, the phenomenon of tumor necrosis and shrinkage is very significant. There is no change in the condition: If imaging shows that the tumor focus of the patient is reduced by less than $50 \%$, the phenomenon of tumor necrosis and shrinkage is not obvious. Disease progression: If imaging shows a $25 \%$ increase in liver cancer lesions, or the formation of new tumor lesions. Effective rate $=($ total number of cases with complete remission and partial remission $) \div$ total number of cases $\times 100 \%$, disease control rate $=$ (total number of cases with complete remission, partial remission and no change in disease condition) $\div$ total number of cases $\times 100 \%$. (2) Occurrence of adverse reactions, including allergy, nausea and vomiting, fever and other adverse reactions. (3) The survival rate of patients followed up for 6 months and 12 months.

\subsection{Statistical methods}

SPSS software package was used for data analysis. T test was used for measurement data, $\chi^{2}$ test was used for counting data, and Cox analysis was performed for prognostic factors. Bilateral $\alpha$ probability $\mathrm{P}$ values were used for statistical inference at all stages. The threshold of statistical significance was 0.05 .

\section{Results}

\subsection{Analysis of recent therapeutic effects}

The effective rate and disease control rate of the TACE group were $38.00 \%$ and $66.00 \%$, the effective rate and disease control rate of the chemotherapy group were $34.00 \%$ and $60.00 \%$, and the effective rate and disease control rate of the observation group were $60.00 \%$ and $84.00 \%$. The effective rate and disease control rate of the observation group were higher than those of the TACE group and chemotherapy group. There was significant difference $(\mathrm{P}<0.05)$. See Table 1 for details. 
Table 1. Recent therapeutic effect of patients in the three groups $(n=50$ cases, $n / \%)$

\begin{tabular}{|c|c|c|c|c|c|c|c|}
\hline Groups & Fully recovered & Partial recovered & No improvement & Disease progress & $\begin{array}{l}\text { Therapeutic } \\
\text { response rate }\end{array}$ & & $\begin{array}{l}\text { Disease contro } \\
\text { rate }\end{array}$ \\
\hline TACE Group & 7 & 9 & 17 & 17 & $16(38.00 \%)$ & $\Delta$ & $33(66.00 \%)$ \\
\hline $\begin{array}{l}\text { Chemotherapy } \\
\text { group }\end{array}$ & 8 & 9 & 13 & 20 & $17(34.00 \%)$ & $\boldsymbol{\Delta}$ & $30(60.00 \%)$ \\
\hline Observation Group & 17 & 13 & 12 & 8 & $30(60.00 \%)$ & & $42(84.00 \%)$ \\
\hline
\end{tabular}

Note: Compared with patients in chemotherapy group, $\triangle \mathrm{P}<0.05$; Compared with patients in observation group, $\boldsymbol{\Delta} \mathrm{P}<0.05$.

\subsection{Analysis of the occurrence of adverse reactions}

The adverse reaction rate of TACE group was $20.00 \%$, chemotherapy group was $42.00 \%$, and observation group was $18.00 \%$. The adverse reaction rate of observation group and TACE group was lower than that of chemotherapy group, and there was a significant difference $(\mathrm{P}<0.05)$. See Table 2 for details.

Table 2. Incidence of adverse reactions in the three groups $(n=50$ cases, $n / \%)$

\begin{tabular}{|c|c|c|c|c|c|}
\hline Groups & Allergy & Nausea and vomiting & Fever & Others & Total \\
\hline TACE Group & 1 & 2 & 5 & 2 & $10(20.00 \%) \triangle$ \\
\hline Observation Group & 1 & 1 & 4 & 3 & $9(18.00 \%)$ \\
\hline
\end{tabular}

Note: Compared with patients in chemotherapy group, $\triangle \mathrm{P}<0.05$; Compared with patients in observation group, $\boldsymbol{\Delta} \mathrm{P}<0.05$.

\subsection{Analysis of survival rate of patients}

The follow-up survival rate in 6-month was $80.00 \%(40 / 50)$ and the 12 -month follow-up survival rate was $50.00 \%$ $(25 / 50)$ in the TACE group, and the 6-month follow-up survival rate was $82.00 \%(41 / 50)$ and the 12-month follow-up survival rate was $48.00 \%(24 / 50)$ in the chemotherapy group. The survival rate of the observation group was $96.00 \%(48 / 50)$ at 6 months follow-up and $74.00 \%(37 / 50)$ at 12 months follow-up. The survival rate of the observation group at 12 months follow-up was higher than that of the TACE group and chemotherapy group, and there was a significant difference $(\mathrm{P}<0.05)$.

\section{Discussion}

The name of liver cancer is strange to Chinese medicine, but it is mostly included in the categories of "abdominal mass, tympanites and jaundice" in the records of related diseases. Some studies ${ }^{[6]}$ have pointed out that such phenomena as deficiency of temper, imbalance of emotion, lingering of toxin accumulation in liver and gallbladder, stagnation of liver Qi and dietary injury exist in the human body simultaneously, which leads to obstruction of Qi and blood stasis in liver. As a consequence, the accumulation and condensation of evil toxins in liver can be developed, which will finally develop into liver cancer. Liver is the hub of rise and fall of Qi, so there is a theory that chronic illness leads to deficiency to give rise to liver disease and spleen disease. According to Traditional Chinese medicine, in order to prolong the survival time of patients with liver cancer and improve their life and health, it is necessary to start with the treatment of specimens and fuzhengquxie. Among them, support is the most critical, which directly affects the survival time of patients.

Huaier grain is the class 1 new drug with the effect of invigorating the spleen, removing blood stasis and activating blood circulation to eliminate diseases, which is suitable for the treatment of blood stasis block and healthy qi weak patients. It can effectively improve the weakness, abdominal distension and pain symptoms of patients, and can be used in the treatment of abdominal pain, fatigue and nagushaoxin induced by liver cancer, breast cancer and lung cancer. Long-term use of the drug can enhance human immunity, improve disease symptoms and stabilize tumor lesions. Combined application of TACE can better control the tumor focus of liver cancer patients, inhibit tumor growth, relieve liver pain, improve the treatment effect and prolong the survival time of patients.

It can be seen from the above that significant effect can be achieved with the combined application of TACE and Huaier granule in the treatment of patients with advanced liver cancer.

\section{Acknowledgments}

This paper is supported by 2017 Zhejiang TCM Science and Technology Planning Project "Clinical study of Huaier granule combined with TACE in the treatment of lung metastasis of liver cancer" (2017ZA032). 


\section{References}

[1] Gao Lidong, Wang Xiumei, Wu Jianliang. Clinical efficacy and safety analysis of percutaneous portal vein puncture combined with lobaplatin for injection in the treatment of advanced hepatocellular carcinoma[J]. Chinese Journal of Primary Medicine and Pharmacy, 2019, 26(16):1970-1976.

[2] Liu Jianfei, Li Feng, Wang Ningfang, et al. Effects of transcatheter arterial chemoembolization combined with radio frequency ablation on survival, liver function and T lymphocyte subgroup in patients with advanced hepatocellular carcinoma[J]. Progress in Modern Biomedicine, 2021, 21(9):1669-1672, 1701.

[3] Jin Dan, Liu Li, Wu Mingyong, et al. Effects of transcatheter arterial chemoembolization and thermoperfusion chemoembolization on liver function in patients with advanced hepatocellular carcinoma[J]. Modern Instruments, 2019, 25(3):56-60.

[4] Zhao Junyan, Lu Yan, Liu Xiaoya. Application of oxaliplatin combined with fluorouracil and pirubicin in the treatment of advanced hepatocellular carcinoma by transcatheter arterial chemoembolization and nursing intervention[J]. Chinese Journal of Rational Drug Use, 2021, 18(4):80-84.

[5] Li Xinyi, Wang Qimei, Deng Xiangsheng, et al. Clinical study of hepatic arterial infusion of Brucea javanica oil emulsion injection combined with lipiodol embolization in the treatment of advanced hepatocellular carcinoma[J]. Hunan Journal of Traditional Chinese Medicine, 2020, 36(8):5-7, 17.

[6] He Chaoxiong, Feng Huigang, Weng Yu. Clinical study of TACE combined with radioactive 125I seed and Huaier granule in the treatment of advanced hepatocellular carcinoma[J]. Chinese Medical Innovations, 2018, 15(6):13-16. 